\title{
I Lost my Patient: Time to Introspect
}

\author{
N. Kannan ${ }^{1}$
}

Received: 27 September 2015 / Accepted: 13 October 2015 /Published online: 17 October 2015

(C) Indian Association of Surgical Oncology 2015

Dear Sir,

I lost my patient.

My 50 year old patient with operable hormone receptor positive early breast cancer refused to undergo surgery on the day of surgery and chose to try herbal medicines. I was aghast. Here was a college teacher choosing something completely and unthinkably unscientific (by my belief) after being counselled for surgery followed by adjuvant therapy.

I spent a week trying to reconcile with the event. She returned a month later and underwent surgery as advised by our multi-disciplinary team.

Cancer care today is in a great flux with a bewildering range of options arising from the wedlock of technology and medicine. No two practitioners offer exactly the same therapy. Varied therapies offer similar outcomes. In this mydriad of competing therapies the patient is often perplexed, confused and overwhelmed with the barrage of information, advise, options while many of us doctors often forget the needs, emotions, wishes of the patient in our zeal to present and use the newest therapy.
We in the practice of "Allopathy" wish to weigh every decision in the light of rigorous scientific enquiry. Scientific practice of medicine demands evidence. At the same time we wish to embark on the path of personalised medicine. The personalization we wish to pay cognisance to, is the molecular signature of disease. But what about the personalization of care to the bodily condition, social status, dietary habits, personal needs, emotional condition, beliefs and aspirations of an individual patient.

The use of Complementary and Alternative medicine is progressively increasing in Integrated Medical Practice. Centuries old wisdom of traditional medicine should not be dismissed summarily. The desire to take recourse to alternative medical therapies possibly stems from their innate appeal to patients.

Why are we surprised when herbal medicine works and evidence based medicine fails? We cannot, in a moment of evidence driven madness summarily dismiss the use of herbal medicine for cancer when we almost always use our grandmothers soup for a sore throat.

This is a time to think and introspect on (the extent and limits of) our knowledge and how we use it.
N. Kannan

majkannan@gmail.com

1 Department of Surgical Oncology, Command Hospital, Pune 411040, India 